EPJ Web of Conferences 80,00016 (2014)

DOI: $10.1051 /$ epj conf/ 20148000016

C) Owned by the authors, published by EDP Sciences, 2014

\title{
Callan-Symanzik approach to infrared Yang-Mills theory
}

\author{
Axel Weber ${ }^{1, a}$ and Pietro Dall'Olio ${ }^{1, b}$ \\ ${ }^{1}$ Instituto de Física y Matemáticas, Universidad Michoacana de San Nicolás de Hidalgo, \\ Edificio C-3, Ciudad Universitaria, A. Postal 2-82, 58040 Morelia, Michoacán, Mexico
}

\begin{abstract}
Dyson-Schwinger equations are the most common tool for the determination of the correlation functions of Landau gauge Yang-Mills theory in the continuum, in particular in the infrared regime. We shall argue that the use of Callan-Symanzik renormalization group equations has distinctive advantages over the Dyson-Schwinger equations, in particular for the vertex functions. We present a generalization of the infrared safe renormalization scheme proposed by Tissier and Wschebor in 2011. The comparison with the existing lattice data for the gluon and ghost propagators can be used to determine the most appropriate renormalization scheme.
\end{abstract}

Dyson-Schwinger equations have given access to the deep infrared (IR) regime of Landau gauge Yang-Mills theory. The first solutions found show a scaling behavior of the propagators in the IR [1]. Several years later, another type of solutions of the same equations was discovered [2], with a massive gluon propagator and a finitely enhanced ghost propagator in the IR (decoupling solutions). To date, Dyson-Schwinger equations are still the main tool for the (semi-)analytical exploration of the IR regime of Yang-Mills theory.

In a parallel development that actually initiated much earlier with Gribov's observation of the existence of gauge copies in his famous 1978 paper [3] and was later worked out in great detail by Zwanziger [4], the theoretical foundations of IR Yang-Mills theory were laid. While this so-called Gribov-Zwanziger scenario seemed to confirm the existence of the scaling solutions, a more recent refinement [5] favors the decoupling type of solutions. Finally, the results of simulations of YangMills theory in the Landau gauge on huge lattices [6] have been interpreted by most workers in the field as confirming the realization of the decoupling type of solutions in three and four space-time dimensions.

In this contribution, we will present a different technique for the exploration of the IR regime of Yang-Mills theory. We intend to reproduce the results of lattice simulations in the Landau gauge that restrict the gauge field configurations to the (first) Gribov region, but make no attempt to reach the fundamental modular region. The restriction to the Gribov region implies the breaking of BRST invariance in the continuum formulation [4]. The most important consequence of the broken BRST invariance for the IR regime is the appearance of a mass term for the gluon field [5]. Indeed, the addition of a gluonic mass term to the Yang-Mills action has been shown to reproduce all the solutions (two types of scaling solutions and the decoupling solution) found before with the help of DysonSchwinger equations when solving the Callan-Symanzik equations for this theory in the IR regime in

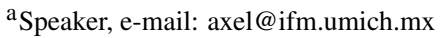

be-mail: pietro@ifm.umich.mx
} 
an epsilon expansion around the upper critical space-time dimension [7]. This analysis also shows that the decoupling solution is the one that is physically realized since it is the only one that corresponds to an IR attractive fixed point (for dimensions above two).

For the rest of this contribution we will focus on the decoupling solution. Tissier and Wschebor [8] have shown that straightforward one-loop perturbation theory applied to Yang-Mills theory with a gluon mass term yields results for the gluon and ghost propagators that fit surprisingly well the propagators found on the lattice in three and four space-time dimensions over the whole range of momenta. It is apparent, however, that a renormalization group improvement is necessary for a quantitative description of the ultraviolet (UV) regime in four space-time dimensions, and very likely also for the IR regime in three dimensions.

Two comments are in order before we move on: first, the BRST invariance of Yang-Mills theory is essential for the construction of the Hilbert space of the theory, the proof of unitarity and also the Kugo-Ojima confinement criterion [9]. It is not clear at present how these constructions could be adapted to the case of broken BRST symmetry which we have assumed here from the outset. We shall have nothing new to say on this matter. The second comment concerns the gluonic mass term: it has been shown in numerous occasions that a gluon propagator of the decoupling type violates spectral positivity (just as in the more obvious case of the scaling type), and it thus does not describe the propagation of a physical particle.

We shall now set up a renormalization scheme for the theory that allows for a consistent formulation of Callan-Symanzik equations, i.e., that avoids the appearance of a Landau pole. Let us remark that we use the term "Callan-Symanzik equations" to refer to the equations that express the invariance of the bare correlation functions with respect to variations of the renormalization scale (not with respect to variations of the mass parameter), in accord with the use of the term in modern textbooks on quantum field theory.

We begin with the Euclidean action of Yang-Mills theory in Landau gauge, including, in addition, a mass term for the gluon field (we then arrive at a Curci-Ferrari model). It is convenient to introduce the Nakanishi-Lautrup auxiliary field $B^{a}$ to implement the gauge condition. Then the action is, in four space-time dimensions,

$$
S=\int d^{4} x\left(\frac{1}{4} F_{\mu \nu}^{a} F_{\mu \nu}^{a}+\frac{1}{2} A_{\mu}^{a} m^{2} A_{\mu}^{a}+\partial_{\mu} \bar{c}^{a} D_{\mu}^{a b} c^{b}+i B^{a} \partial_{\mu} A_{\mu}^{a}\right) .
$$

Note that there are two (bare) parameters in the theory, the gluon mass parameter $m$ and the coupling constant $g$ (that appears in the covariant derivative $D_{\mu}^{a b}$ and in the field strength tensor $F_{\mu \nu}^{a}$ ). The mass parameter $m$ can be thought of as representing the Gribov parameter $\gamma$ that is introduced in order to implement the restriction to the Gribov region [3]. The Gribov parameter, and as a consequence the mass parameter, is in principle fixed through the horizon condition [4] in terms of the unique scale of the theory, $\Lambda_{\mathrm{QCD}}$ (equivalently, in terms of the value of $g$ at an arbitrary scale). Since the exact relation between $m$ and $\Lambda_{\mathrm{QCD}}$ is not known, at least not beyond the lowest perturbative order, we treat $m$ here as an independent parameter that has to be fixed by comparison with the "experiment" (lattice simulations), just as the mass of a physical particle in a theory without confinement.

We renormalize the theory through the conditions (in momentum space)

$$
\begin{aligned}
& \left.\Gamma_{A A}^{T}(p,-q)\right|_{p^{2}=\mu^{2}}=\left(\mu^{2}+m^{2}\right)(2 \pi)^{D} \delta(p-q), \\
& \left.\Gamma_{A A}^{L}(p,-q)\right|_{p^{2}=\mu^{2}}=m^{2}(2 \pi)^{D} \delta(p-q), \\
& \left.\Gamma_{c \bar{c}}(p,-q)\right|_{p^{2}=\mu^{2}}=\mu^{2}(2 \pi)^{D} \delta(p-q),
\end{aligned}
$$



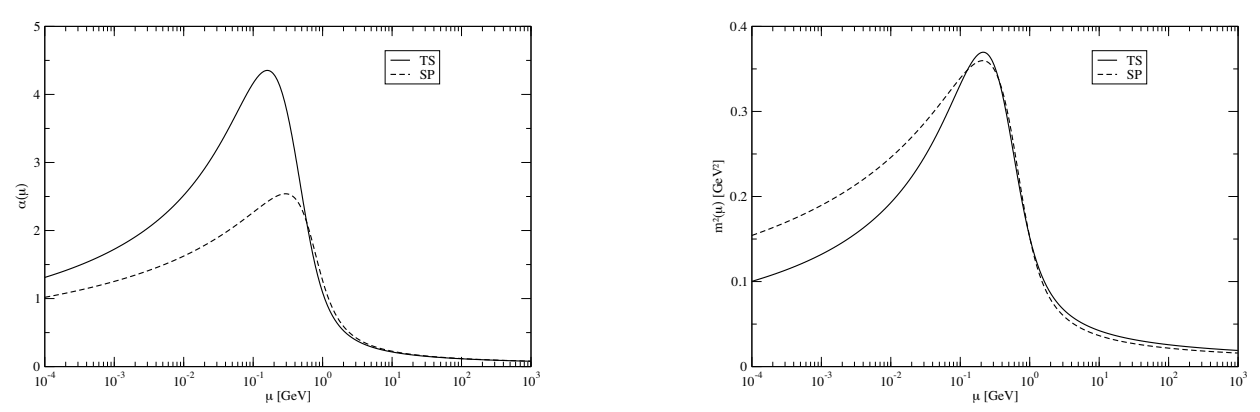

Figure 1. Running fine structure constant (to the left) and running mass parameter (to the right) in the Taylor scheme (TS) vs. the symmetry point scheme (SP).

which determine the field renormalization constants $Z_{A}$ and $Z_{c}$ and the mass parameter $m$ at the renormalization scale $\mu$. The symbols $\Gamma_{A A}^{T}$ and $\Gamma_{A A}^{L}$ refer to the transverse and longitudinal part, respectively, of the proper gluonic two-point function. All two-point functions carry the trivial color factor $\delta^{a b}$ because of global color symmetry.

The normalization conditions (2) were first proposed by Tissier and Wschebor in Ref. [10], where they were written in a different, but equivalent form. For the definition of the coupling constant, Tissier and Wschebor use the renormalized ghost-gluon vertex in the Taylor limit where the ghost momentum vanishes. In this limit, the quantum corrections to the vertex vanish exactly [11] which makes the calculation of the beta function in this renormalization scheme particularly simple.

For a first assessment of the renormalization scheme dependence we propose to consider the ghostgluon vertex at the symmetry point for the definition of the renormalized coupling constant, instead of using the Taylor limit. The calculation of the beta function in this scheme involves an explicit evaluation of the one-loop corrections to the ghost-gluon vertex (at the symmetry point). In Fig. 1 we compare the running of the strong fine structure constant $\alpha(\mu)=g^{2}(\mu) / 4 \pi$ and the square of the mass parameter $m^{2}(\mu)$ as functions of the renormalization scale, obtained from the integration of the corresponding beta functions, in the two renormalization schemes Although the qualitative behavior of the curves is the same in both schemes, a strong quantitative dependence on the renormalization scheme is found. This is an indication that there are important contributions from two-loop diagrams or from other vertex functions that have not been taken into account.

We should like to clarify that at any fixed order of perturbation theory, the results for all correlation functions have to be exactly the same in any renormalization scheme (except for possible constant rescalings of the fields, and assuming that physically equivalent values of the parameters $g$ and $m$ are chosen in all schemes). However, the results of applying Callan-Symanzik equations to different renormalization schemes can be essentially different since they sum up parts of higher-loop diagrams by "extrapolating" the information obtained from the correlation functions to a given loop order in different ways.

Notice from Fig. 1 that the running coupling constant (or the fine structure constant) tends to zero logarithmically in the large-momentum limit as it should (asymptotic freedom). The same is true for the mass parameter. However, in the beta functions it is really the dimensionless combination $\mathrm{m}^{2} / \mu^{2}$ that matters, so the UV limit of a massless and BRST-invariant theory is actually reached much faster than logarithmically. 
Interestingly, both the coupling constant and the mass parameter also tend logarithmically to zero in the IR limit of zero momentum. In the case of the coupling constant, this means that the IR fixed point is trivial, too. However, the fact that the mass parameter tends to zero does not mean that one recovers a massless theory in the deep IR: the scale dependence of the gluon field renormalization constant leads to a finite limit of the gluon propagator at zero momentum. The trivial IR fixed point is thus essentially different from the trivial UV fixed point.

Let us now compare the results for the gluon and ghost propagators obtained in the two different renormalization schemes via the integration of the corresponding Callan-Symanzik equations with the data of lattice simulations. To this end, we have to determine those values of the parameters $g$ and $m$ at some (arbitrary) scale that lead to the best possible fits to the lattice data. The actual values of these parameters will be different for the two renormalization schemes. Our strategy is to first fix the coupling constant (or, equivalently, the scale $\Lambda_{\mathrm{QCD}}$ ) in the far UV where the influence of the mass parameter is negligible, and in a second step determine an appropriate value of the mass parameter such as to adjust the propagators to the lattice data in the mid- and low-momentum regimes. In this way, we reproduce the well-known UV behavior of the theory and obtain a real assessment of the degree to which the renormalization scheme is able to fit the data in the IR.

In practice, the quality of the fit of the coupling constant is limited by the relatively low precision of the lattice data for the ghost dressing function in the UV which partially compromises our fitting strategy (the dressing function is the ratio of the full propagator to the tree-level propagator). In addition, our results are renormalization group improvements of one-loop results, while it is wellknown that for a reliable fit to the UV data renormalization group improved two-loop results are needed.

In Figs. 2 and 3 we show the best fits to the lattice data we have obtained with the definition of the renormalized coupling constant in the Taylor limit and at the symmetry point of the ghost-gluon vertex, respectively. The results obtained from the integration of the Callan-Symanzik equations in Fig. 2 are very similar in the IR regime to the ones found by Peláez, Tissier and Wschebor in Ref. [13], but our fit to the lattice data is better in the UV regime. It is apparent that a very good fit to the ghost dressing function data can be achieved in the Taylor scheme, and a very good fit to the gluon propagator on the lattice is obtained by using the symmetry point. When assessing the quality of the fits, one should keep in mind that the flow functions of the Callan-Symanzik equations have only been determined to one-loop precision, so that a perfect fit to the data can certainly not be expected. In any case, we have not been able to produce satisfactory fits to both propagators at the same time in either of the two renormalization schemes. Again, it would appear that important contributions from two-loop diagrams or from other vertex functions are missing.

Now, it is already apparent in the IR renormalization group analysis of Ref. [7] that the renormalized coupling constants associated with the ghost-gluon, the three-gluon and the four-gluon vertices, respectively, behave very differently in the IR, which is yet another manifestation of the broken BRST symmetry. We are thus compelled to formally consider (at least) three different coupling constants in the theory, for each of which we determine a beta function from the calculation of the corresponding quantum corrections. Since BRST symmetry has to be recovered in the limit of large momenta, the three renormalized coupling constants must become equal and the running mass parameter must vanish in this limit, which technically represents a fine-tuning condition. This condition relates the values of the three renormalized coupling constants, so that we still have only two independent parameters in the theory (the mass parameter and one of the coupling constants).

As a first step, we assume that the renormalized coupling constant extracted from the four-gluon vertex is approximately equal to the one corresponding to the three-gluon vertex, but we treat the latter coupling constant as independent of the one associated with the ghost-gluon vertex which we 

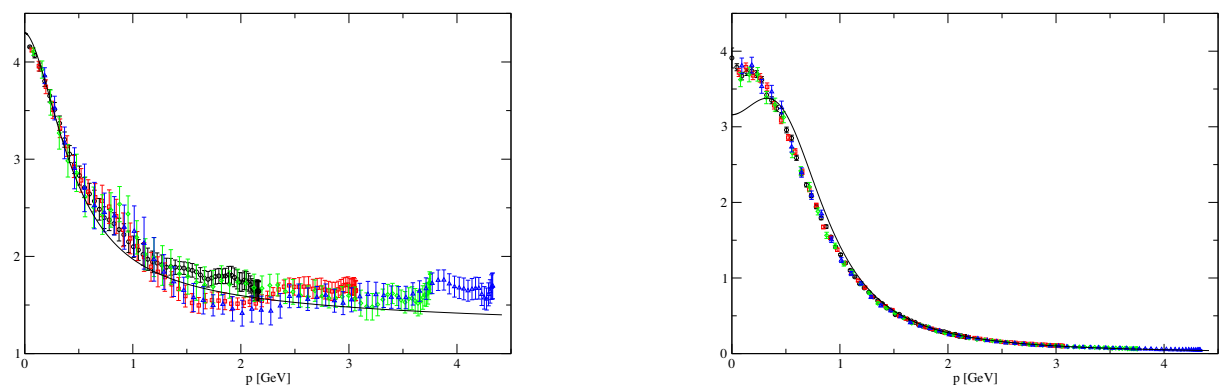

Figure 2. Ghost dressing function (to the left) and gluon propagator function (to the right) in the Taylor scheme compared to the lattice results of Ref. [12]. The different colors of the lattice data visible in the online version correspond to different directions on the lattice.
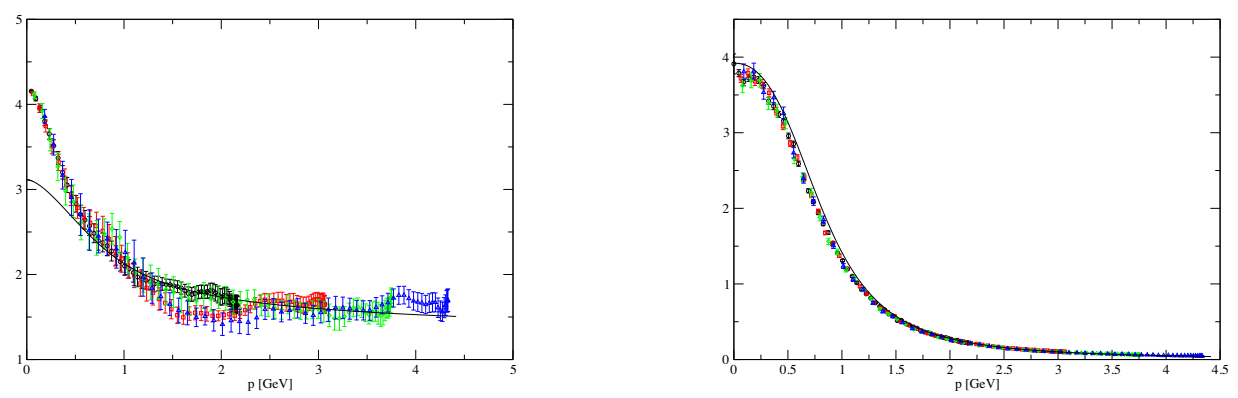

Figure 3. Ghost dressing function (to the left) and gluon propagator function (to the right) in the symmetry point scheme compared to the lattice results.

have been focussing on so far (both the three-gluon and the ghost-gluon vertices are evaluated at the symmetry point for the definition of the respective coupling constants). After integrating the coupled differential equations for these two coupling constants and the mass parameter, we can then solve the Callan-Symanzik equations for the propagators. Again, the fit of the resulting propagators to the lattice data is not quite satisfactory, even considering that the flow functions are determined only at the one-loop level.

We shall close this contribution by mentioning the advantages of applying Callan-Symanzik renormalization group equations (as compared to the use of Dyson-Schwinger equations, or functional renormalization group equations for that matter): the scheme presented here can be systematically improved by going to higher loop orders and including further coupling constants (there is only a finite number of relevant and marginal couplings with respect to the IR and/or the UV fixed points). The calculation of the flow functions is analytical (the evaluation of some of the appearing Feynman diagrams may require numerical integration, but at least the IR and UV limits can usually be extracted analytically), and the renormalization group equations constitute a set of coupled ordinary first-order differential equations which can be handled numerically without too much effort. There are, in any 
case, only two parameters to be fixed in order to define the theory. Different (consistent) renormalization schemes can be devised. An optimal scheme can in principle be determined by comparing with the lattice data (and tuning the two free parameters).

The advantages of employing Callan-Symanzik equations are clearest when it comes to determining the vertex functions: there is no need to guess at the most important structures or to construct ansätze for the vertex functions, everything is in principle determined (to the order considered) by the corresponding Feynman diagrams, compare, e.g., Ref. [13] with [14]. As an example, in our calculation with two different coupling constants described above we immediately find for the IR behavior of the renormalized coupling constant $g_{3}(\mu)$ associated with the (completely transverse) three-gluon vertex (to be precise, with the tensor structure of the tree-level vertex which gives the dominant contribution in the UV as well as in the IR limit) that

$$
g_{3}(\mu)=\left(\frac{6 \pi^{2}}{N}\right)^{1 / 2} \frac{\ln \left(\mu^{2} / \Lambda_{1}^{2}\right)}{\left[\ln \left(\Lambda_{0}^{2} / \mu^{2}\right)\right]^{3 / 2}},
$$

where $\Lambda_{0}$ and $\Lambda_{1}$ are two different scales relevant to the IR behavior (ultimately determined in terms of $\Lambda_{\mathrm{QCD}}$ ) with $\Lambda_{1}<\Lambda_{0}$, and $N$ is the number of colors. Equation (3) is valid for $\mu^{2} \ll \Lambda_{0}^{2}$ and shows that $g_{3}(\mu)$ passes through zero at $\mu=\Lambda_{1}$. Taking into account the IR behavior of the gluon field renormalization constant, Eq. (3) implies that the three-gluon vertex function (for the tree-level tensor structure) at the symmetry point $p^{2}=q^{2}=r^{2}$ is proportional to $\ln \left(p^{2} / \Lambda_{1}^{2}\right)$ in the IR, which is compatible with the findings of Refs. [13, 14].

\section{Acknowledgements}

The authors would like to thank Attilio Cucchieri and Tereza Mendes for allowing them to use their lattice data, and Chris Stephens for interesting discussions. Support by Conacyt project CB-2009/131787 and CIC-UMSNH is gratefully acknowledged.

\section{References}

[1] L. von Smekal, A. Hauck and R. Alkofer, Phys. Rev. Lett. 79, 3591 (1997); C. Fischer and R. Alkofer, Phys. Lett. B 536, 177 (2002)

[2] A.C. Aguilar and A.A. Natale, JHEP0408, 057 (2004); P. Boucaud, T. Brüntjen, J.P. Leroy et al., JHEP0606, 001 (2006); M. Frasca, Phys. Lett. B 670, 73 (2008)

[3] V.N. Gribov, Nucl. Phys. B 139, 1 (1978)

[4] D. Zwanziger, Nucl. Phys. B 323, 513 (1989); Nucl. Phys. B 399, 477 (1993)

[5] D. Dudal, J.A. Gracey, S.P. Sorella et al., Phys. Rev. D 78, 065047 (2008)

[6] I.L. Bogolubsky, E.M. Ilgenfritz, M. Müller-Preussker and A. Sternbeck, Proc. Sci. LAT2007, 290 (2007); A. Cucchieri and T. Mendes, Proc. Sci. LAT2007, 297 (2007); A. Sternbeck, L. von Smekal, D.B. Leinweber and A.G. Williams, Proc. Sci. LAT2007, 340 (2007)

[7] A. Weber, Phys. Rev. D 85, 125006 (2012)

[8] M. Tissier and N. Wschebor, Phys. Rev. D 82, 101701 (2010)

[9] T. Kugo and I. Ojima, Prog. Theor. Phys. Suppl. 66, 1 (1979)

[10] M. Tissier and N. Wschebor, Phys. Rev. D 84, 045018 (2011)

[11] J.C. Taylor, Nucl. Phys. B 33, 436 (1971)

[12] A. Cucchieri and T. Mendes, Proc. Sci. QCD-TNT09, 026 (2010)

[13] M. Peláez, M. Tissier and N. Wschebor, Phys. Rev. D 88, 125003 (2013)

[14] A.L. Blum, M.Q. Huber, M. Mitter and L. von Smekal, Phys. Rev. D 89, 061703 (2014) 